INVESTIGACIÓN/RESEARCH

\title{
PROGRAMACIÓN RADIOFÓNICA EN CUBA. UN MODELO SINGULAR EN LA ERA DE INTERNET
}

Michel D. Suárez Sian'1. Universidad Complutense de Madrid. España. michelds@ucm.es

\section{RESUMEN}

Este trabajo analiza las características de la programación radiofónica en Cuba, a partir de las tres emisoras nacionales generalistas y de la visión de un grupo de radialistas. En una primera etapa (1922-1960), la radio cubana representó una de las experiencias más exitosas de América Latina. La segunda, que transcurre entre 1960 y la actualidad, ha estado marcada por un cambio radical en la titularidad de las emisoras, al igual que en los procesos vinculados a la modernización programática. Esta investigación se enfoca en las particularidades del modelo de programación vigente, su interrelación con el ecosistema de medios de comunicación y el grado de influencia de las nuevas tecnologías en los cambios. Para ello aplica un análisis de contenido y entrevistas a expertos. El artículo intenta demostrar el impasse que padece la radio cubana, derivado de factores políticos, tecnológicos y conceptuales.

PALABRAS CLAVE: Radio - Programación - Cuba - Creatividad - Internet.

\footnotetext{
${ }^{1}$ Autor correspondiente:

Michel D. Suárez Sian: Departamento de Periodismo II de la Universidad Complutense de Madrid. España.

Correo: michelds@ucm.es
} 


\title{
CUBAN RADIO PROGRAMMING. A SINGULAR MODEL IN THE INTERNET ERA
}

\begin{abstract}
This paper analyzes the characteristics of radio programming in Cuba, from the three national generalist broadcasters. In the first stage (1922-1960), the Cuban radio represented one of the most successful in Latin America. The second time between 1960 and the present, has been marked by a radical change in ownership of the radio stations, as in the processes related to the modernization program. This research focuses on the specifics of the current programming model, his relationship with the media ecosystem and the degree of influence of new technologies on the changes. In this paper we used content analysis and expert interviews. The article focuses on the impasse of Cuban radio caused by certains political, technological and conceptual factors.
\end{abstract}

KEYWORDS: Radio - Programming - Cuba - Creativity - Internet.

\section{INTRODUCCIÓN}

La programación radiofónica es uno de los campos menos estudiados en las Ciencias de la Información. Desde el punto de vista bibliográfico, la radio es un ámbito escasamente analizado, lo cual se refleja en su pobre volumen de producción y en el déficit de investigaciones realizadas (Rodero, 2009). En general, la radio cubana padece las mismas carencias científicas, pese a su relevancia en dicha sociedad (Fonseca, 2011).

En la primera mitad del siglo $\mathrm{XX}$, la radio cubana protagoniza un acelerado desarrollo tecnológico y formal. Experimenta amplios márgenes de aceptación social, e incluso, en la década de 1940, consigue exportar guiones y programas a otros países latinoamericanos. La primera transmisión radiofónica cubana se produce el 22 de agosto de 1922, a través de la emisora 2LC. Otros hitos aparecen sucesivamente con las emisoras CMQ y RHC Cadena Azul, muy competitivas entre sí y responsables de la creación de los programas más escuchados de la época.

En 1948, Félix B. Caignet escribe la primera radionovela, El derecho de nacer (López, 1998), que hace historia en Cuba y en otros países. En 1931 comienza a emitirse La Voz del Aire, el primer radioperiódico organizado integralmente para ser transmitido (López e Infante, 2007), y en 1937 "el espectáculo más oído de todos los tiempos", Chan Li Po, escrito por Caignet, "el primero de continuidad seriado" en Cuba y la América hispana (López e Infante, 2007). A punto de iniciarse la década de los 50, en 1947, surge Radio Reloj, considerado el primer canal de información continua del mundo (López, 1998). Historiadores ideológicamente opuestos coinciden en la trascendencia de los aportes radiofónicos de la época, aunque discrepan en las causas y en los efectos. 
López e Infante (2007), ambos funcionarios gubernamentales, consideran que los avances cubanos no resultaron fortuitos porque el país estaba "sujeto a la dominación neocolonial de los Estados Unidos". Al factor geopolítico, condicionado por la "pujante expansión de los consorcios eléctricos del vecino del Norte", adicionan la situación de La Habana de entonces, "con su desbordante mundo de espectáculos públicos". Es decir, una mezcla entre transferencia tecnológica directa y efervescencia cultural. Sin embargo, las circunstancias políticas derivadas del triunfo de la revolución cubana expropiaciones de emisoras, censura y eliminación de la iniciativa privada - conducen a la caída del imperio radiofónico (Suárez, 2006). En 1960, el Gobierno cubano nacionaliza las 66 cadenas del país y crea el Frente Independiente de Emisoras Libres (López e Infante, 2007). Dos años más tarde, se funda el Instituto Cubano de Radiodifusión (ICR), que centraliza la gestión y la política editorial. En 2013, el sistema cubano cuenta con 99 emisoras, seis de ellas nacionales. Todas bajo control estatal. Sus empleados son cerca de 7.000, "cuya misión es ofrecer una programación radial portadora de altas virtudes político-ideológicas, sociales, éticas y estéticas, en concordancia con la batalla de ideas que libra Cuba" (Radio Cubana, 2013).

La radio ocupa una posición relevante en el ecosistema cubano de medios de comunicación, debido a las carencias de la televisión. En Cuba solo existen cinco cadenas nacionales de televisión en abierto, y los canales de pago solo pueden ser contratados por extranjeros y diplomáticos. Tampoco existe conexión a internet desde los hogares, salvo excepciones (Suárez, 2012). En dicho contexto, la radio cubana conserva contenidos y formatos que la televisión cubre más eficientemente en otros países, desde los años 70 .

\subsection{Creatividad y programación radiofónica.}

Las parrillas radiofónicas han sufrido transformaciones radicales desde que Tommy Cowan, empleado de la empresa de electrodomésticos Westinghouse, se convirtió en 1921 en el "primer programador" (Contreras y Palacio, 2003), al pedir prestado un fonógrafo a Thomas Edison para la apertura formal de la WJZ en Nueva Jersey (Barnouw, 1966).

Las definiciones de programación radiofónica, alusivas a una organización u orden, han resistido el paso del tiempo. Sin embargo, la forma y el contenido han experimentado severas transformaciones desde principios del siglo XX. La programación radiofónica es la planificación de una relación comunicativa entre una empresa de radio y una audiencia, mediante unos contenidos sistematizados y organizados en un conjunto armónico, según unos criterios de selección, dosificación y ordenación (Cebrián Herreros, 2001). O simplemente la previsión de los programas que van a ser emitidos durante un tiempo determinado a través de una emisora de radio (Muñoz y Gil, 1994).

La programación radiofónica no debe entenderse como un ente aislado o un producto de laboratorio. Diversos factores obligan a su revisión periódica (Merayo, 2003), entre ellos las preferencias de los oyentes, las exigencias publicitarias, las variaciones en las 
plantillas, los acontecimientos informativos y la programación de la competencia. Faus (1995) resume el tema de manera ejemplar: el arte de la programación descansa, sobre todo, en la auscultación permanente de la vida y la sociedad.

Cebrián Herreros (2007, pp. 160-161) afirma que la programación, en tanto proceso comunicativo, "trata de atender los comportamientos sociológicos de la audiencia, adapta los contenidos a sus necesidades y situaciones laborales y de ocio". Dichas funciones han eliminado contenidos anteriores, "especialmente los más creativos, como los documentales y los de ficción". Según dicha visión, las tendencias programáticas se han orientado hacia la cobertura de la actualidad como un valor intrínseco de la radio. "La orientación informativa de la radio generalista no le deja tiempo para el sosiego creativo, para la reelaboración".

Las comparaciones entre la radio actual y la predominante en el siglo XX generan debates entre los investigadores que se dedican al tema. Asuntos como la creatividad, aparentemente perdida, o la prevalencia de formatos noticiosos e interactivos, que excluyen a dramatizados y temáticos, ocupan espacio en los estudios contemporáneos sobre radiodifusión. En este sentido, Rodero (2005) cuestiona que la radio de hoy ignore los contenidos y las fórmulas más creativas de presentación, para favorecer la información de actualidad, presentada de la forma más sencilla y, si puede ser, siempre en directo, es decir, de la manera más económica y fácil posible:

La radio se ha transformado en el eterno parlante, muchas veces sin sentido, sin preparación, ni rigor, lo que obliga al oyente a escuchar opiniones sin fundamento, palabras, palabras y más palabras. La radio se ha convertido en monotonía, en improvisación, pura realidad, en un medio que ya no obliga a sentir. Esta ausencia de creatividad ya se está pagando en forma de escasa audiencia infantil y juvenil (Rodero, 2005, p. 135).

Desde el punto de vista de la radio en Cuba, Garcés (2007) estima que "la televisión ha recuperado mucho del terreno perdido y ocupa un lugar cada vez más notorio como fuente de educación, entretenimiento e información". Según expone, en Cuba, como en otras partes del mundo, las audiencias jóvenes tienden a ser más audiovisuales:

Traer los oyentes a la radio (...) y llevar la radio a los oyentes (...), depende más de la voluntad de los realizadores que de la inversión de cuantiosos recursos. Probablemente, la naturaleza crecientemente competitiva del entorno mediático cubano devele para muchas emisoras la necesidad de articular estrategias de socialización más agresivas, en lugar de esperar porque nuevos oyentes 'caigan' dentro del círculo de sus receptores habituales.

Pero, en un escenario extremadamente competitivo - donde conviven otros medios e internet-, ¿cuánta radio se escucha actualmente en el mundo? Según López Vigil (2000), más que en los años 50, pero ahora también se ve más televisión, más películas (aunque no sea en el cine), se habla más por teléfono y se navega más en internet. Cada medio tiene su originalidad, su espacio, sus ventajas y limitaciones, con margen para la coexistencia. "La radio le cambió el libreto a la prensa. La televisión se lo cambió a la radio. Y hoy, la globalización de la cultura y la revolución tecnológica se lo ha cambiado a todos los medios de comunicación". 
Cuba enfrenta una situación peculiar: todas las emisoras pertenecen al Gobierno. "Son de propiedad estatal o social y no pueden ser objeto, en ningún caso, de propiedad privada, lo que asegura su uso al servicio exclusivo del pueblo trabajador y del interés de la sociedad" (Constitución de la República de Cuba, 1992). Por tanto, la política de programación, los conceptos editoriales y operativos y las inversiones emanan exclusivamente de decisiones centralizadas.

Los historiadores oficiales defienden el monopolio estatal - implantado en 1960como garantía de una programación subvencionada, sin presiones comerciales y, por consiguiente, más innovadora y de mayor calidad (López e Infante, 2007). Sin embargo, en su análisis sobre las radios privadas y públicas iberoamericanas, Merayo (2007, p. 20) asegura que la comercial ha demostrado ser más imaginativa en la invención de nuevos formatos y programas, obligada por la necesidad de competir y sobrevivir en un mercado cada vez más complejo. El investigador español no encuentra ningún mal en ello, "pues fue la radio comercial la primera que encontró, en la creación de cadenas, una eficaz fórmula de rentabilizar costes y aumentar audiencias y coberturas".

En las últimas cuatro décadas, la radio ha conseguido reinventarse frente a otros medios, más allá del aspecto tecnológico. También...

...influyó decisivamente la rápida y eficaz reacción de muchos de sus profesionales que, lejos de aferrarse en actitud suicida a unos planteamientos superados por la competencia de la televisión, supieron buscar y encontrar el hueco que la radio tenía reservado en exclusiva y llenarlo con nuevas ideas y nuevos formatos inexplorados hasta entonces (Carcedo, 2011).

En este sentido, Fraticelli (2011, p. 126) pone en duda que el desarrollo de la ficción televisiva haya fulminado al drama radiofónico. De acuerdo con su tesis, incluso antes de que apareciera la televisión, los radioteatros no eran muy valorados por la crítica cultural, porque la radio popular no alcanzaba a ser considerada como artística. "Todo lo contrario sucedía con las ficciones televisivas (...) Cada transposición de un programa de la radio a la televisión era festejada por los metadiscursos del momento". Tobi (2007) apunta a la década de los años sesenta como la etapa donde se configura un momento de reorientación del medio: "Como en un juego de postas, el mito de la radio como servicio viene a reemplazar al mito de la radio como agente cultural". El compromiso de la radio "no es ya para con la Nación, vinculada al ámbito de la cultura o de la industria - como en los 30-, sino con el oyente individualizado, al que busca informar y entretener". Entonces lo masivo se articula con lo particular y emerge "una radio para cada oyente" (Tobi, 2007).

¿Propuso la radio una nueva relación con sus oyentes cuando apareció la televisión?, pregunta Fraticelli (2011). Y, atendiendo a los tipos discursivos, su respuesta es afirmativa: sí hubo un cambio profundo en la relación de la radio con su público. El nuevo oyente ya no era convocado para relacionarse con una manifestación artística, como un concierto o una obra de ficción:

Los tipos discursivos que se expandieron en la programación propusieron otro pacto 
enunciativo poniendo en contacto al oyente con un real que se desarrollaba en el mismo momento que él estaba escuchando (la competencia de un concurso, la transmisión de un encuentro deportivo, la información de un acontecimiento de actualidad) o con música grabada, mayormente en estudio (Fraticelli, 2011, p. 127).

A pesar de los profundos cambios discursivos y de recepción, algunas corrientes reivindicativas confían en recuperar funciones y esquemas de la era pre-audiovisual. Rodero (2005, p. 145) espera que las nuevas tecnologías - radio digital y en internetpuedan propiciar un cambio donde los comunicadores radiofónicos del futuro produzcan contenidos más creativos, entre ellos los géneros de ficción. En su opinión, el objetivo siempre consistirá en "seguir ahondando en las posibilidades expresivas de la radio, no para desvelar por completo su misterio, sino para continuar creándolo", pues "la creatividad es lo único que la mantendrá viva".

En otra cuerda, Cebrián Herreros (2007, p. 21-22) asegura que la radio se encuentra en una encrucijada nueva: ahora trata de hacer frente a la eclosión mediática producida por la innovación técnica, y a la incorporación de todo ello dentro de la sociedad de la información. Internet es sin dudas uno de los grandes activos de la radio actual, debido a la filosofía participativa con que se gestiona el medio y a la diversidad de opciones individuales de entretenimiento e información:

Esta nueva situación posibilita al oyente la autogestión de la propuesta radiofónica, y sitúa a la audiencia dentro de un escenario de tiempos paralelos - lineal y no linealdonde se diferencian los contenidos individuales - asincrónicos- y la parrilla tradicional - sincrónica - , que deja de tutelar la única estrategia - vertical y lineal de consumo (Murelaga, 2007, p. 123).

Mientras se produce este reacomodo, la radio resiste. En un país como Estados Unidos, con una potente industria audiovisual, la radio conserva una fuerte presencia en el entorno mediático. En 2011, nueve de cada diez estadounidenses mayores de 12 años (93\%) había utilizado o tenía una radio AM/FM, una caída de sólo tres puntos porcentuales desde 2001. Estos datos sitúan a la radio convencional en segundo lugar, solo superada por la televisión, como el medio más frecuente en la vida de la gente (State of the Media, 2012). En España, la penetración de la radio en 2012 fue del 61,9\%, la cifra más alta desde 1997. Los oyentes de entre 25 y 34 años representan el mayor grupo etáreo, con el 69\% (Estudio General de Medios, 2012).

\section{METODOLOGÍA}

El sistema cubano de radiodifusión cuenta con seis emisoras nacionales, de las cuales tres podrían considerarse especializadas: Radio Reloj (información continua), CMBF (música clásica) y Radio Enciclopedia (música instrumental). Las otras tres - Radio Rebelde, Radio Progreso y Radio Taíno - responden, según las premisas de este trabajo, a un esquema generalista y constituyen el objeto de la investigación. El análisis de sus parrillas pretende definir la tipología programática, así como los atributos formales y conceptuales. Por tales razones elegimos el análisis de contenido, un proceso donde las características relevantes del mensaje se transforman en unidades que permiten su descripción y examen por medio de la codificación (Hernández 
Sampieri et. al, 2003).

A través del análisis de contenido también pueden formularse inferencias reproducibles que puedan aplicarse a su contexto, a partir de ciertos datos, con una orientación fundamentalmente empírica, exploratoria, vinculada a fenómenos reales y de finalidad predictiva (Krippendorff, 1990).

La muestra seleccionada - parrillas de Radio Progreso, Radio Taíno y Radio Rebelde- comprende las emisiones de lunes a viernes, en el caso de las dos primeras, y de martes a viernes, en el de la última. La decisión responde a las características de cada semana-tipo, en la que, al completarse la frecuencia semanal, las muestras asumen una suficiente representación del total. El análisis - aplicado entre el 25 y el 29 de marzo de 2013 - se basa en los estilos de programación propuestos por López Vigil (2000), aunque adaptados al entorno investigado: mosaico de programas (espacios de treinta minutos como promedio diario), bloques moderados (entre $60 \mathrm{y}$ 120 minutos promedio) y bloques extensos (más de 120 minutos).

Los ítems establecidos para el estudio fueron:

a) Número de programas diarios

b) Duración promedio

c) Tendencia programática: mosaico de programas, bloques moderados o extensos

d) Formatos y programas: Informativos, musicales y magazines de entretenimiento, dramatizados y otros

Adicionalmente, las entrevistas a expertos buscan delimitar las condiciones generales de la programación en Cuba, sus problemas y posibles soluciones. Las entrevistas, una de las técnicas más utilizadas en investigaciones sobre comunicación, pretenden conocer la opinión de personas informadas o con experiencias relevantes para el estudio (Rojas, 2002). En el caso que nos ocupa, cumplen un objetivo auxiliar, complementan el análisis de contenido y ayudan a diagnosticar la situación estudiada. Hemos seleccionado la modalidad de entrevista cerrada (Gaitán y Piñuel, 1998), para recabar opiniones, de modo estandarizado y estructurado, sobre el estado programático de la radio cubana.

Las entrevistas se realizaron a 30 sujetos informados (expertos), entre ellos periodistas, productores o presentadores de la radio cubana, en la actualidad o anteriormente. Mediante un muestreo no probabilístico, se seleccionaron -deliberada y explícitamente - entrevistados que facilitaran datos muy específicos sobre lo que se pretendía estudiar (Gaitán y Piñuel, 1998). La mayoría de los consultados son licenciados (63\%) o doctores (13\%), y el 70\% tiene más de diez años de experiencia en los medios. La edad predominante oscila entre los 26 y 45 años (el $70 \%$ de los entrevistados). Las entrevistas se aplicaron entre el 16 de febrero y el 2 de marzo de 2013, teniendo en cuenta los siguientes aspectos generales:

a) Tipo de programación predominante en la radio cubana

b) Metodología de elaboración de las parrillas 
c) Capacidad de actualización conceptual

d) Propuestas y soluciones a considerar

e) Situación de géneros y formatos ya obsoletos en otras realidades

f) Principales problemas de la radio cubana

\section{ANÁLISIS Y DISCUSIÓN}

El análisis de contenido examinó las parrillas de Radio Progreso, Radio Rebelde y Radio Taíno, las tres emisoras nacionales generalistas. Radio Progreso presenta la mayor cantidad de programas diarios del conjunto (56), para un promedio de 25,7 minutos por programa, de lunes a viernes (Tabla I). Sus espacios ni siquiera alcanzan la media hora promedio, por lo que la parrilla se corresponde con la clasificación de "mosaico extremo". La fragmentación es una de sus principales características, junto a la emisión de dramáticos y unitarios temáticos, un enfoque cada vez más en desuso en la radiodifusión. Los informativos representan el 17,9\%, los musicales y magazines de entretenimiento, el 58,4\%; los dramatizados, el 14\%; y otros programas (educativos, culturales), el 9,7\% (Tabla II). Se manifiesta una propensión hacia los espacios de entre 15 y 35 minutos (casi la mitad de la parrilla) y hacia los de menos de 10 minutos (más de un tercio). La excepción son los contenedores de 118 minutos o más, cuya representación se limita al 30\% del tiempo total diario.

Radio Rebelde, por su parte, presenta la menor cantidad de programas diarios del conjunto, con 14, para un promedio de 102,8 minutos por programa, de martes a viernes (Tabla I). La parrilla está concebida a través de bloques, aunque en su manifestación más moderada. A diferencia de la emisora anterior, los informativos (incluyendo los espacios de información deportiva) y los musicales y magazines de entretenimiento constituyen la base del esquema. No existen programas dramatizados ni de otro tipo, que no sean los mencionados. Tres de sus programas, de 180 minutos per cápita, y uno, de 240 minutos, aseguran una mayoría horaria del 54,16\% en cada jornada. Otros dos, de 150 minutos per cápita, abarcan el 20,83\%. Estos programas de entre 240 y 150 minutos - cubren el 74,99\% del tiempo diario. El predominio informativo alcanza el 54,16\% del total (Tabla II). Aunque su tendencia es al uso de franjas horarias, no excluye los programas de 15 y 30 minutos.

La tercera cadena nacional generalista, Radio Taíno, exhibe un esquema de 20 programas diarios (de lunes a viernes) y representa una opción intermedia entre las dos anteriores (Tabla I). Su parrilla está diseñada a través de bloques moderados y el promedio de minutos por programa es de 72. Seis de sus espacios duran 120 minutos per cápita y uno, 180. Dicha suma representa el 62,5\% de la jornada. Además, otros cinco programas alcanzan los 60 minutos (el 20,83\% del tiempo total). Los informativos cubren el $14,58 \%$ de la programación, con predominio para musicales y magazines de entretenimiento, el 79,17\% (Tabla II). No existen dramatizados, aunque sí otros programas temáticos, entre ellos culturales y científico-técnicos (6,25\%). 
Tabla 1. Número de programas diarios, según el tiempo.

\begin{tabular}{|l|c|c|c|c|c|c|c|}
\hline & \multicolumn{9}{|c|}{} \\
\hline HORQUILLA & $\mathbf{- 1 0}$ & $\mathbf{1 5 - 3 5}$ & $\mathbf{4 5 - 6 0}$ & $\mathbf{8 0 - 1 2 0}$ & $\mathbf{1 4 0 - 1 8 0}$ & $\mathbf{+ 1 8 0}$ & Total \\
\hline & & & & & & & \\
\hline Radio Progreso & 21 & 27 & 4 & 2 & 2 & 0 & 56 \\
\hline Radio Rebelde & 0 & 5 & 2 & 1 & 5 & 1 & 14 \\
\hline Radio Taíno & 0 & 8 & 5 & 6 & 1 & 0 & 20 \\
\hline & & & & & & & \\
\hline TOTAL & 21 & 40 & 11 & 9 & 8 & 1 & 90 \\
\hline
\end{tabular}

Fuente: Elaboración propia.

Tabla 2. Formatos y géneros. Porcentaje diario.

\begin{tabular}{|l|c|c|c|c|}
\hline & Informativos & $\begin{array}{c}\text { Musicales y } \\
\text { magazines de } \\
\text { entretenimiento }\end{array}$ & Dramatizados & Otros \\
\hline Radio Progreso & $17,9 \%$ & $58,4 \%$ & $14 \%$ & $9,7 \%$ \\
\hline Radio Rebelde & $54,16 \%$ & $45,84 \%$ & 0 & 0 \\
\hline Radio Taíno & $14,58 \%$ & $79,17 \%$ & 0 & $6,25 \%$ \\
\hline
\end{tabular}

Fuente: Elaboración propia.

Las entrevistas complementan la información del análisis de contenido y exponen la visión de expertos que trabajan o trabajaron en la radio cubana. Una sólida mayoría $(97 \%)$ considera que el tipo de programación predominante es generalista, entendida como aquella que mezcla información, música, deportes, entretenimiento y dramatizados. El 69\% revela que la parrilla se elabora sobre la base de orientaciones gubernamentales, el 14\% con investigaciones de audiencia y el 10\% teniendo en cuenta otros factores (Gráfico I). En este último acápite aparecen las ideas del personal de las emisoras. 
Gráfico 1. Fuentes para la elaboración de la programación.

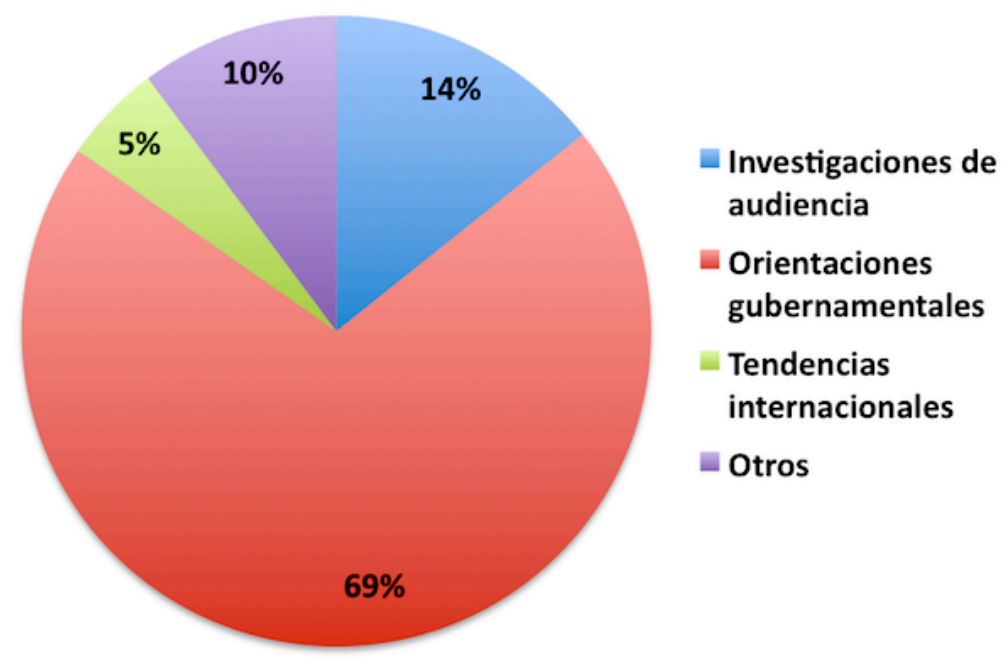

Fuente: Elaboración propia.

Los elementos que deberían sopesarse en el diseño de la programación se manifiestan muy repartidos (pregunta multi-respuesta), aunque el 38\% cita las necesidades del auditorio como el más importante. Luego, y por ese orden, las tendencias internacionales (29\%) y el impacto de otros medios, como la televisión e internet $(26 \%)$. En este caso, las necesidades del Gobierno solo fueron mencionadas por el $4 \%$ de los entrevistados (Gráfico II). Esta opinión contrasta con el peso de las orientaciones políticas en la confección de las parrillas, como muestra el Gráfico I.

\section{Gráfico 2. ¿Cómo debería elaborarse la programación?}

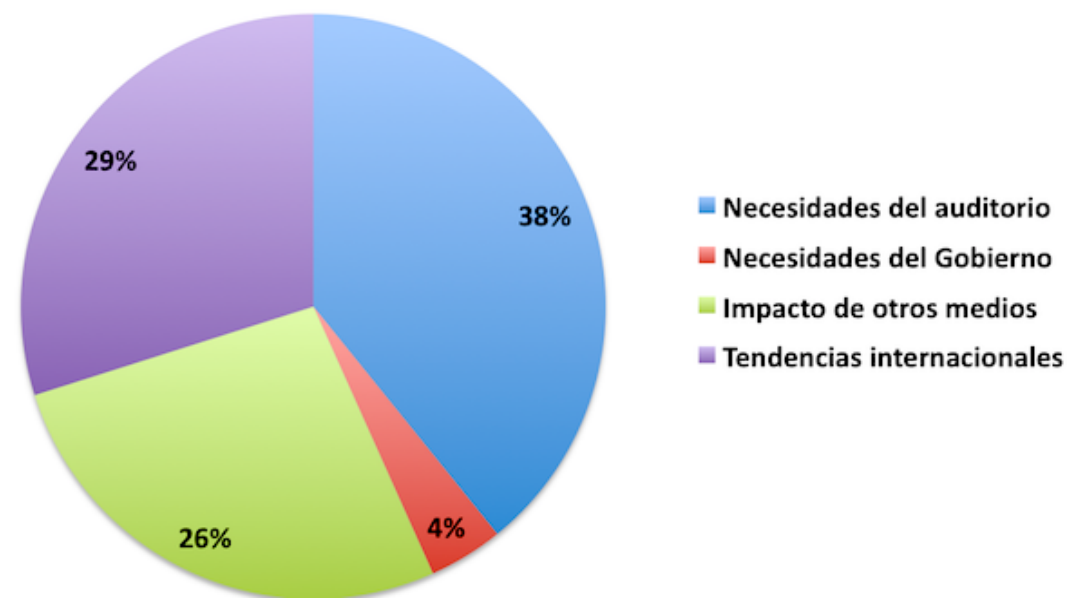

Fuente: Elaboración propia.

Los entrevistados dejan escaso margen para la duda en su calificación de la programación cubana: el 87\% cree que está "desfasada", frente al 13\% que la valora de "actualizada". Sin embargo, otras respuestas revelan incertidumbre sobre las posibles soluciones. Una mayoría del $43 \%$ apuesta por disminuir el tiempo de los programas 
para crecer en cantidad; es decir, obrar en dirección contraria a los modelos internacionales predominantes y acentuar el esquema cubano de las últimas décadas. El 37\% cree lo contrario, que debería aumentarse el tiempo de duración de los programas para que estos ocupen las principales franjas horarias. Un $20 \%$ cita otros criterios temporales para elaborar la programación, entre ellos que la duración atienda al destinatario y al entorno, así como una mayor y mejor especialización de los contenidos (Gráfico III).

\section{Gráfico 3: Posibles soluciones.}

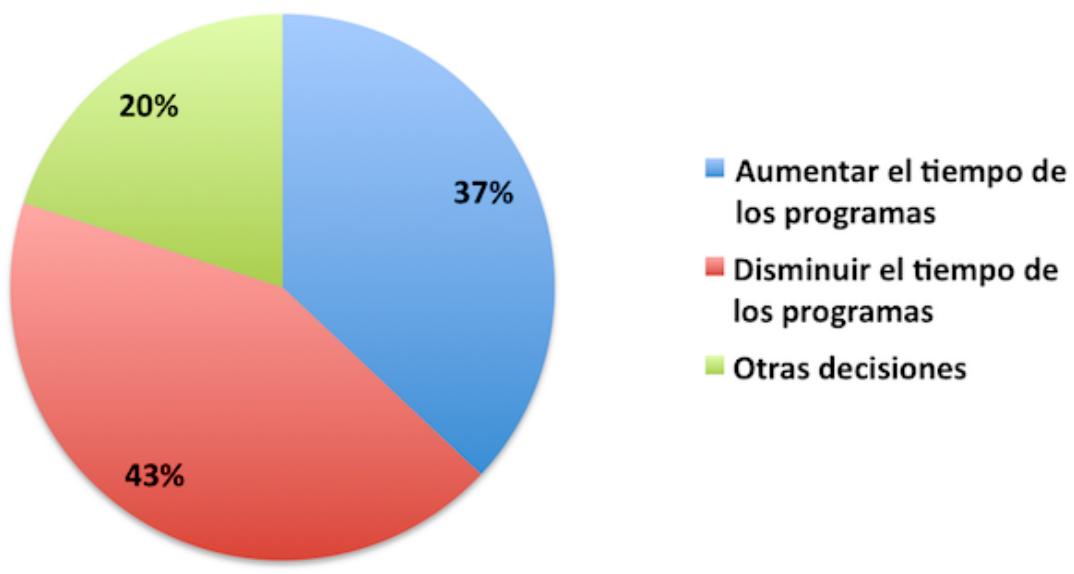

Fuente: Elaboración propia.

Otros datos de las entrevistas refuerzan la situación de excepcionalidad. En cuanto a los posibles criterios genéricos a tener en cuenta, el 50\% considera que las emisoras deberían seguir mezclando información y música y el 40\% que deberían especializarse en informativas o musicales. En resumen, una mayoría rechaza el modelo especializado. Otro resultado llamativo se produce en torno a la continuidad de los espacios dramatizados. El 63\% cree que deberían mantenerse y el 30\%, lo contrario. En cuanto a la permanencia de los programas infantiles, el dato de aprobación es aún mayor: $67 \%$. Una posible explicación a ambas respuestas emerge en lo adelante: el $45 \%$ de los entrevistados afirma que aún existen espacios infantiles y dramáticos por la debilidad del cine y la televisión. La decisión parece circunstancial, aunque el 38\% opina que tal situación responde al apoyo de la audiencia. Un 15\% indica otras posibilidades, entre ellas que "en muchos hogares aún la radio constituye un medio comunicativo fuerte". Además, millones de hogares carecen de ordenadores o reproductores de DVD, "por lo que la radio se complementa con la televisión". 


\section{Gráfico 4: ¿Por qué se emiten aún infantiles y dramatizados?}

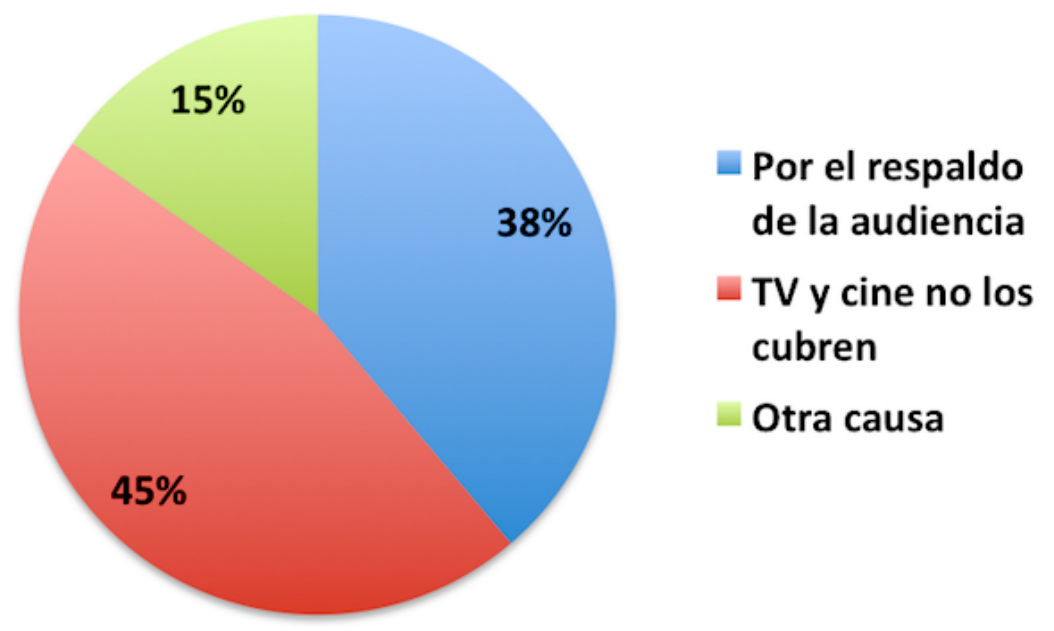

Fuente: Elaboración propia.

La última pregunta de las entrevistas pedía identificar el principal problema de la radio cubana, entre tres opciones. El 70\% de los entrevistados apunta a los problemas políticos, el $20 \%$ a los económicos y el $10 \%$ a la formación y al talento del personal. Debido al tipo de propiedad imperante en los medios (100\% estatal), las relaciones de poder y la verticalidad del sistema de radiodifusión, el dato podría interpretarse como muy relevante para entender la situación general de la radio cubana.

\section{CONCLUSIONES}

Los resultados han sido analizados con perspectiva histórica y en un contexto internacional de profundas transformaciones comunicativas. Esto permite plantear que la radio cubana afronta una parálisis compleja, derivada del concepto programático predominante, las relaciones de propiedad, el ecosistema local de medios de comunicación, el aislamiento tecnológico y los factores políticos.

En cuanto al concepto, las tres emisoras nacionales generalistas tienden a estructurar la programación en bloques moderados, salvo Radio Progreso, que conserva un esquema de mosaico ultrafragmentado. Dicho modelo pretende producir una oferta heterogénea, para amplios públicos, desde el punto de vista social, etáreo y cultural. Su filosofía está cada vez más alejada de las prácticas comunicativas internacionales, donde los medios buscan nichos específicos de mercado para fidelizarlos y atenderlos mejor.

En los otros dos casos se manifiesta una mayor presencia de bloques y una mejor definición de las propuestas, según su público potencial, pero todavía lejos de las tendencias proclives a segmentar las audiencias y a especializar las ofertas. Un resultado preocupante es la confianza depositada en el modelo informativo-musical como posible solución al desfase. Tal perspectiva contribuye a la conservación del target totalizador. Otra realidad constatada es el elevado número de programas diarios, que conspira contra una racional concentración de los recursos humanos y 
técnicos. En este sentido, resulta inquietante la apuesta por la disminución del tiempo de los programas, un camino que consolidaría el modelo mosaico en su variante más exagerada.

En otros países, la evolución de las parrillas se ha visto condicionada, constantemente, por las transformaciones del mercado publicitario. Sin embargo, esto no ha podido suceder en Cuba porque su modelo radiofónico no depende de la publicidad. Tampoco la eclosión de las nuevas tecnologías de la información parece haber influido en el diseño de las programaciones. La ausencia de internet en los hogares prácticamente hace de Cuba un país desconectado. Evidentemente, la inexistencia de amenazas e interacciones, desde otros ámbitos comunicativos, funciona como cortafuegos para la modernización. En ausencia de servicios a la carta, podcasts, streaming y de otros medios competitivos, la parrilla vertical pervive sin demasiadas presiones para el cambio. Ni siquiera se ha producido una readecuación a partir del impacto de la televisión: ante los problemas de la industria audiovisual cubana, la radio perdura como escenario natural de formatos dramáticos e infantiles.

Los resultados también revelan una alta incidencia gubernamental en las decisiones programáticas. De hecho, una contundente mayoría considera que el principal problema de la radio cubana es de índole política. Si se compara la tendencia innovadora exhibida hasta 1960 con el estancamiento de 2013, podría establecerse una relación causa-efecto negativa entre la monopolización estatal del sector y los resultados actuales. Frente al escenario tecnológico, cultural y de consumo, presente en el entorno natural de Cuba, los investigadores sociales deberían profundizar en las perspectivas de la radio local.

\section{BIBLIOGRAFÍA}

Barnouw, E. (1966): A History of Broadcasting in the United States: A tower in Babel; to 1933. Oxford: Oxford University Press.

Carcedo, D. (2011): "El presente y el futuro de la radio". En: Ortiz, M. A.; López, N. (editores) Radio 3.0. Una nueva radio para una nueva era. Madrid: Editorial Fragua.

Cebrián Herreros, M. (2007): Modelos de radio, desarrollos e innovaciones. Del diálogo y la participación a la interactividad. Madrid: Editorial Fragua.

Cebrián Herreros, M. (2001): Información radiofónica. Mediación técnica, tratamiento y programación. Madrid: Síntesis.

Contreras, J.M.; Palacio, M. (2003): La programación de televisión. Editorial Síntesis, Madrid.

Constitución de la República de Cuba (1992): Constitución de la República de Cuba. Artículo 53. La Habana. Disponible en: www.cuba.cu/gobierno/cuba.htm. Consultado el 24 de enero de 2013. 
Estudio General de Medios (2012): "Resumen general EGM, febrero-noviembre de 2012". Asociación para la Investigación de Medios de Comunicación, Madrid. Disponible en: www.aimc.es/-Datos-EGM-Resumen-General-.html. Consultado el 1 de febrero de 2013.

Faus Belau, A. (1995): "Los caminos de la radio. Orientaciones del medio en Europa", en revista Telos No. 42, Junio - Agosto. Madrid: Fundación Telefónica. Disponible en: http://sociedadinformacion.fundacion.telefonica.com/telos/anteriores/num_042/in dex_042.html?cuaderno_central9.html. Consultado el 28 de enero de 2013.

Fonseca Muñoz, B.E. (2011): "Las rutinas productivas en la programación dramatizada de Radioarte", en revista Razón y Palabra No. 77, Agosto-Octubre 2011. Instituto Tecnológico de Monterrey. Disponible en: www.razonypalabra.org.mx/varia/77\%202a\%20parte/30_Fonseca_V77.pdf. Consultado el 22 de enero de 2013.

Fraticelli, D. (2011): "Los cambios de la programación radiofónica cuando aparece la televisión", en La Trama de la Comunicación, Volumen 15. Universidad Nacional de Rosario. Rosario: UNR Editora.

Gaitán, J.A. y Piñuel, J.L. (1998): Técnicas de investigación en comunicación social. Elaboración y registro de datos. Madrid: Editorial Síntesis.

Garcés, R. (2007): "La crisis de los discursos radiofónicos", en Revista Latina de Comunicación Social, 62, pp. 227-232. Disponible en: www.revistalatinacs.org/200719RaulGarces.htm. Consultado el 11 de marzo de 2013. DOI: 10.4185/RLCS-62-2007-745-227-232.

Hernández Sampieri, R. et. al (2003): Metodología de la investigación. México DF: McGraw-Hill Interamericana.

Krippendorff, K. (1990): Metodología de análisis de contenido. Teoría y práctica. Barcelona: Paidós.

López, O. e Infante, R. (2007): "La radio en Cuba". En: Merayo, A. (coord.) La radio en Iberoamérica. Evolución, diagnóstico y prospectiva. pp. 175-176. Sevilla: Comunicación Social Ediciones y Publicaciones.

López, O. (1998): La radio en Cuba. La Habana: Editorial Pueblo y Educación, segunda edición.

López Vigil, J.I. (2000): Manual urgente para radialistas apasionados. La Habana: Editorial Pablo de la Torriente Brau.

Merayo, A. (2007): "La estimulante diversidad de la radio iberoamericana". En: Merayo, A. (coord.) La radio en Iberoamérica. Evolución, diagnóstico y prospectiva. p. 20. Sevilla: Comunicación Social Ediciones y Publicaciones. 
Merayo, A. (2003): Para entender la radio. Salamanca: Publicaciones Universidad Pontificia de Salamanca.

Muñoz, J.J. y Gil, C. (1994): La Radio: Teoría y Práctica. Madrid: Instituto Oficial de Radio Televisión Española.

Murelaga Ibarra, J. (2007): "Reflexiones sobre la transformación del concepto 'programación radiofónica'. De la parrilla al mensaje", en Palabra Clave. Volumen 10. No. 2. Diciembre, Cundinamarca: Universidad de La Sabana.

Radio Cubana (2013): "Misión de la Radio Cubana". Instituto Cubano de Radio y Televisión. La Habana. Disponible en: www.radiocubana.cu/index.php/faqspreguntas-mas-frecuentes/31-quienes-somos/27-mision-de-la-radio-cubana.

Consultado el 25 de febrero de 2013.

Rodero Antón, E. (2009): "Y siempre, por último, con ustedes: la radio. Revisión de la producción bibliográfica y hemerográfica radiofónica en España", en revista Admira No. 1. Universidad de Sevilla. Disponible en:

www.academia.edu/363011/Y_siempre_por_ultimo_con_ustedes_la_radio._Revision _de_la_produccion_bibliografica_y_hemerografica_radiofonica_en_Espana.

Consultado el 21 de enero de 2013.

Rodero Antón, E. (2005): "Recuperar la creatividad radiofónica. Razones para apostar por la radio de ficción", en revista Anàlisi No. 32, pp. 133-146, Barcelona: Disponible en: http://ddd.uab.es/pub/analisi/02112175n32p133.pdf. Consultado el 27 de enero de 2013.

Rojas Soriano, R. (2002): Investigación social: Teoría y praxis. México DF: Editorial Plaza y Valdés.

State of the media (2012): "Audio: How Far Will Digital Go?". Pew Research Center for the People \& the Press. Disponible en: http://stateofthemedia.org/2012/audio-howfar-will-digital-go. Consultado el 18 de diciembre de 2012.

Suárez, M. D. (2012): "Cuba: Internet, acceso y sociedad del conocimiento", en revista Razón y Palabra No. 81. Noviembre. Instituto Tecnológico de Monterrey. Disponible en: www.razonypalabra.org.mx/N/N81/M81/07_Suarez_M81.pdf. Consultado el 9 de diciembre de 2012.

Suárez, M. D. (2006): "Cuba: medio siglo de no-periodismo", en Revista Hispano Cubana No. 25 Primavera-verano, Madrid. pp. 68-80.

Tobi, X. (2007): "La radio en los 60: redefiniciones a partir de la llegada de la TV", en revista Question No. 13. Universidad Nacional de La Plata, Argentina. Disponible en: http://perio.unlp.edu.ar/question/numeros_anteriores/numero_anterior13/nivel2/e ditorial.htm. Consultado el 26 de febrero de 2013. 


\section{Michel D. Suárez Sian}

Doctor en Ciencias de la Información por la Universidad Complutense de Madrid y Licenciado en Periodismo por la Universidad de Oriente (Cuba). Líneas de investigación: información en radio y televisión, narrativa audiovisual y nuevas tecnologías de la información. Autor de los libros "Dramaturgia audiovisual", "Canales de noticias en televisión" y "Son de la Loma". Trabaja como periodista y colabora en el Departamento de Periodismo II de la Universidad Complutense de Madrid. 